\title{
Updates to UK emergency and recovery advice following changes in international guidance
}

\author{
A. F. NISBET, S. M. HAYWOOD, K. MORTIMER, J. R. SIMMONDS
}

\begin{abstract}
HPA is updating and consolidating its advice on radiation emergencies and recovery. Current advice was published in 1997 . Since 2007, the ICRP has issued a set of recommendations to elaborate its guidance for emergency exposure and existing exposure situations. It is expected that the European Basic Safety Standards, when published, will also reflect the ICRP recommendations. The new ICRP guidance represents a marked change in approach, with emphasis placed on optimisation of whole protection strategies using reference levels of residual dose. These new concepts as well as the relevant lessons identified following the Fukushima accident will be included in the new UK advice document. The scope of the UK advice includes reactor and transport accidents as well as releases from waste stores, reprocessing and defence activities. The revised advice will consider the initiation of emergency countermeasures based on averted dose criteria and optimisation of the subsequent protection strategy based on reference levels of residual dose. The advice will illustrate that the type of protection strategy selected depends on the contribution of different exposure pathways over time to projected dose, and this will vary according to the scenarios considered as reasonably foreseeable. Due to the potential impact of the advice, a wide range of stakeholders are being consulted. In particular, feedback will be required on the potential for adapting current practices for sheltering and stable iodine prophylaxis to situations involving longer duration releases or those with a prolonged threat phase. The advice document will contain guidance for emergency planning and response, criteria for the withdrawal of emergency countermeasures, factors to consider during the transition to an existing exposure situation and the management of long term contaminated areas. It is the first time that the whole spectrum of advice will be presented in a single publication, which is expected to be published in 2013, following a public consultation process.
\end{abstract}

Health Protection Agency, Centre for Radiation, Chemical and Environmental Hazards, Chilton, Didcot, Oxon, OX11 0RQ, UK. 